Original paper

\title{
Expression of vascular endothelial growth factor $A$ in liver tissues of infants with biliary atresia
}

\author{
Alif Allam¹, Mohammed El-Guindi', Hatem Konsowa', Dina El. Azab', Maha Allam¹, Tahany Salem¹, \\ Haidy Mohammed Zakaria \\ ${ }^{1}$ National Liver Institute, Menoufia University, Shebin El-koom, Menoufia, Egypt \\ ${ }^{2}$ Quesna Central Hospital, Menoufia, Egypt
}

\begin{abstract}
Aim of the study: Assessment of hepatic expression of vascular endothelial growth factor A (VEGF-A) in liver tissues of infants with biliary atresia (BA).

Material and methods: This retrospective study included 35 infants with BA (BA group), and 38 infants with cholestasis due to causes other than BA (non-BA group). All patients had undergone full history taking, through clinical examination, routine investigations and immunostaining of liver tissue for VEGF-A and cytokeratin 7 (CK7). The diagnosis of BA was confirmed by intraoperative cholangiography. In the non-BA group, other specific laboratory tests according to the expected etiology were done.

Results: Most of the BA group showed positive VEGF-A expression with variable degrees in both bile ducts (BDs; $80 \%$ ), and arterial walls (AWs; $77.2 \%$ ), while most of the non-BA group showed negative staining of VEGF in both BDs and AWs (89.5\% and $86.8 \%$ respectively) $(p<0.0001)$. Positive VEGF expression in the portal structures in both BDs and AWs had $84.9 \%$ and $82.19 \%$ accuracy; respectively. The majority of BA group showed either grade II of positive cytokeratin-7 expression in liver tissues (45.7\%) or grade III (34.3\%), while most of the non-BA group showed grade I $(71.1 \%)(p<0.0001)$. Positive CK7 expression in $>25 \%$ of the liver tissues had $80.8 \%$ accuracy in discriminating between $B A$ and non-BA.
\end{abstract}

Conclusions: VEGF-A expression in the portal structures in liver tissues in both BDs and AWs had very good accuracy in discriminating between BA and non-BA patients.

Key words: growth factor, biliary atresia, cytokeratin.

\section{Address for correspondence}

Haidy Mohammed Zakaria, MD, Quesna Central Hospital, Menoufia, Egypt, phone: +20 1005768306,

e-mail: drhaydi2000@gmail.com

\section{Introduction}

Biliary atresia (BA) encompasses a cholangiopathy of elusive nature affecting extra- and intrahepatic bile ducts, which generally results in cirrhosis irrespective of timely provision of portoenterostomy [1]. Cholangiopathies show specific features with reference to blood vessels because bile ducts are nourished exclusively through the peribiliary vascular plexus formed by hepatic artery branches. Abnormal peribiliary vascular plexus in some diseases is attributable to an ischemic cholangiopathy [2].
The formation of new blood vessels is a typical feature of chronic liver diseases including liver fibrosis, regardless of the underlying etiology. Angiogenesis is believed to promote fibrosis, mostly due to the observation that angiogenesis and fibrotic changes occur simultaneously in many organs including the liver [3]. Vascular endothelial growth factor (VEGF) is an important proangiogenic factor. It acts as a link between angiogenesis, the immune system and tissue remodeling. Expression of VEGF is enhanced in angiogenesis [4].

In $\mathrm{BA}$, there is portal expansion, and the mechanical distortion over vascular structures may induce 
hypoxia/ischemia. Oxygen plays a central role in regulating angiogenesis and VEGF, whenever induced by hypoxia, giving rise to new vessels aiming at restituting adequate tissue oxygenation [5].

Angiogenesis and the expression of vascular factors (such as VEGF) are intimately involved in the pathogenesis of biliary tract diseases. Studies are needed to address the role that VEGF plays in aberrant angiogenesis during biliary fibrosis and how cholangiocytes interact with other liver cell types such as hepatic stellate cells (HSC) and vascular endothelial cells. Manipulation of the signaling mechanisms that regulate angiogenesis may thus represent a new approach to treat cholangiopathies [6].

The aim of this study was to investigate VEGF-A in liver tissues of infants with BA.

\section{Material and methods}

\section{Study population}

This is a retrospective study including 73 infants with neonatal cholestasis in whom liver biopsy was done for etiological diagnosis. All were attendants of the Pediatric Hepatology Department, National Liver Institute, Menoufia University.

After revising the final diagnosis, they were divided into two groups: Group I, 35 infants with biliary atresia (BA group); and Group II, 38 infants with cholestasis due to causes other than BA (non-BA group). Paraffin blocks of liver biopsy of selected patients were retrieved from the archives of the Pathology Department. The study was approved by the Research Ethics Committee of the National Liver Institute, Menoufia University, Egypt.

\section{Etiological diagnosis}

After reevaluation of data of these infants (full history taking, clinical examination and routine investigations) the diagnosis of BA was confirmed by intraoperative cholangiography (IOC) prior to Kasai operation.

The diagnoses of non-BA infants in this study were as follows: progressive familial intrahepatic cholestasis (PFIC) I: 6 patients, PFIC II: 5 patients, PFIC III: 6 patients, cytomegalovirus (CMV) hepatitis: 6 patients, idiopathic neonatal hepatitis: 4 patients, galactosemia: 2 patients, Niemann-Pick type C: 2 patients, congenital hepatic fibrosis: 2 male patients, tyrosinemia: 1 patient, bile acid synthetic defects: 1 patient. Arthrogryposis, renal tubular dysfunction, cholestasis (ARC) syndrome: one patient. Sepsis: one patient.
The revised investigations of the patients included: liver function tests [total and direct bilirubin, total proteins, serum albumin, alanine transaminase (ALT), aspartate transaminase (AST), alkaline phosphatase (ALP), $\gamma$-glutamyl transferase (GGT), prothrombin time and concentration], complete blood count (CBC), screening for TORCH (Toxoplasma, rubella, cytomegalovirus, herpes simplex and others such as septic screen including C-reactive protein (CRP) and pancultures) and hepatotropic viruses as hepatitis $C$ virus antibody ( $\mathrm{HCV}-\mathrm{Ab})$, hepatitis $\mathrm{B}$ virus surface antigen (HBsAg), hepatitis B virus core antibodies ( $\mathrm{HBcIgM}$ ) and (HBcIgG), abdominal ultrasonography with color Doppler, duodenal tube aspiration (DTA), liver biopsy. In the non-BA group, other specific laboratory tests according to the expected etiology were seen.

\section{Histopathological processing and immunostaining of liver tissues in the studied groups}

The slides obtained were processed for staining and immunohistological assessment for: hematoxylin and eosin (H\&E), Masson trichrome, Perls' stain, orcein and periodic acid Schiff for routine histopathological evaluation, immunostaining of VEGF-A, anti-muscle actin, and cytokeratin 7 (CK7), measurement of medial thickening and diameter of the hepatic artery and to calculate the ratio between diameter of the hepatic artery and its medial thickening (REMD).

Positive immunostaining was identified by discoloration of the cytoplasm and/or cell membrane according to each antibody marker used in the study (anti-VEGF; Mouse monoclonal antibody, Ab68334, Abcam, Cambridge, UK, positive control: Human liver carcinoma) and (anti-CK7, OV-TL 12/30 Monoclonal antibody, Lab Vision Corporation, Fremont, USA, positive control: Pancreas/bladder cancer) using high-power fields of view $(400 \times)$ using an Olympus Light Microscope BX43 made in Japan.

Vascular endothelial growth factor (VEGF): VEGF-A immune-staining was assessed semi-quantitatively based on its extent in bile ducts, wall of arterial blood vessels and hepatocytes in high-power fields $(\mathrm{HPF}) \times 400$ using an Olympus Microscope. Adequate expression pattern for the markers was analyzed according to the protocol developed by $\mathrm{Wu}$ et al. as follows: $0=$ no staining, Grade $1=$ light yellow staining, Grade 2 = dark yellow staining, and Grade 3 = brown staining with granular distribution [7].

Cytokeratin 7: CK7 is a marker of biliary epithelium in outlining biliary structures. Ductular reaction was assessed semi-quantitatively based on its extent 
around the limiting plate in high-power fields (HPF) $\times 400$ using Olympus Microscope. Ductular reaction was graded semiquantitatively according to Yabushita et al. as follows: grade 1: CK7 positivity in bile ducts and ductular reaction only, grade 2: CK7 expression in the cytoplasm of periportal hepatocytes in addition to bile ductular reaction, grade 3: CK7 expression in the cytoplasm of not only periportal but intralobular hepatocytes as well in addition to bile ductular reaction; and grade 4: diffuse CK7-positive staining in the cytoplasm of the majority of hepatocytes in addition to bile duct and ductular epithelium [8].

\section{Statistical methods}

Statistical analysis was done using SPSS version 18 on an IBM compatible computer. Descriptive results were expressed as mean \pm standard deviation (mean $\pm \mathrm{SD}$ ) or number $(n)$ and percentage (\%) of individuals with a condition. For quantitative data, statistical significance was tested by applying the Mann-Whitney $U$ test or Student's $t$-test as appropriate. For qualitative data, significance between groups was tested by chisquare test or Fisher's exact test, as appropriate. Correlation was tested by Spearman test. The diagnostic performance was measured as sensitivity, specificity, positive predictive value (PPV) and negative predictive value (NPV) and expressed as a percentage. The cutoff for optimal clinical performance was determined by the receiver operator characteristic (ROC) curve. Results were considered significant if $p$-value $\leq 0.05$.

\section{Results}

The study included 35 infants with BA, and 38 infants with non-BA cholestasis recruited during the period from May 2016 to February 2018. The gender and age at admission were comparable in both groups (BA; $62.48 \pm 22.59$ day and non-BA; $78.02 \pm 48.63)(p>0.05)$ despite the significantly earlier appearance of jaundice in the BA patients (5.88 \pm 7.35 day) than the non-BA group (25.13 \pm 24.34 day) $(p<0.05)$.

The laboratory parameters showed no statistically significant difference between the BA and non-BA group except for ALT, GGT, prothrombin concentration $\%$, and serum ferritin (Table 1).

The ultrasonography features were remarkable between BA and non-BA. Most of the BA group had abnormal GB contractility, a positive TC sign and hepatic subcapsular flow. All BA cases that underwent DTA had no bile (100\%) (Table 2).

The presence of periductular neutrophils $(p<0.001)$, mononuclear inflammatory cells in the ducts and pseudorosette formation $(p<0.05)$ in liver tissues were significantly more frequent in the BA group than in the non-BA group. Also the BA group had portal cellular

Table 1. Liver function tests (LFTs) in the studied groups

\begin{tabular}{|c|c|c|c|}
\hline \multirow[t]{2}{*}{ Parameter } & $\begin{array}{c}\text { BA } \\
(n=35)\end{array}$ & $\begin{array}{l}\text { Non-BA } \\
(n=38)\end{array}$ & \multirow[t]{2}{*}{$P$-value } \\
\hline & Mean \pm SD & Mean $\pm S D$ & \\
\hline $\mathrm{TB}(\mathrm{mg} / \mathrm{dl})$ & $11.5 \pm 3.9$ & $10.35 \pm 4.83$ & 0.128 \\
\hline $\mathrm{DB}(\mathrm{mg} / \mathrm{dl})$ & $8.56 \pm 2.89$ & $7.41 \pm 3.32$ & 0.088 \\
\hline $\mathrm{TP}(\mathrm{g} / \mathrm{dl})$ & $5.45 \pm 0.65$ & $5.38 \pm 1.0$ & 0.398 \\
\hline Albumin (g/dl) & $3.37 \pm 0.45$ & $3.39 \pm 0.7$ & 0.614 \\
\hline AST (U/l) & $212.71 \pm 148.28$ & $321.62 \pm 262.83$ & 0.151 \\
\hline $\mathrm{ALT}(\mathrm{U} / \mathrm{l})$ & $101.66 \pm 70.32$ & $178.11 \pm 146.84$ & 0.03 \\
\hline $\mathrm{ALP}(\mathrm{U} / \mathrm{I})$ & $550.03 \pm 233.23$ & $522.97 \pm 341.5$ & 0.189 \\
\hline GGT (U/I) & $858.5 \pm 494.48$ & $293.68 \pm 486.43$ & 0.0005 \\
\hline Prothrombin time $(s)$ & $12.17 \pm 1.29$ & $13.05 \pm 1.97$ & 0.066 \\
\hline Prothrombin concentration $\%$ & $92.7 \pm 13.32$ & $84.7 \pm 19.34$ & 0.048 \\
\hline Hemoglobin (g/dl) & $9.73 \pm 0.99$ & $10.23 \pm 1.68$ & 0.118 \\
\hline White blood cells $\left(\times 10^{3} / \mu \mathrm{l}\right)$ & $11.53 \pm 3.59$ & $12.38 \pm 4.33$ & 0.547 \\
\hline Platelets $\left(\times 10^{3} / \mu \mathrm{l}\right)$ & $461.7 \pm 191.5$ & $370.9 \pm 180.3$ & 0.066 \\
\hline Ferritin (ng/ml) & $787 \pm 537$ & $2330 \pm 3169$ & 0.037 \\
\hline$\alpha$-fetoprotein (ng/ml) & $8127 \pm 11252$ & $69245 \pm 168893$ & 0.052 \\
\hline
\end{tabular}

TB - total bilirubin, DB - direct bilirubin, TP - total protein, AST - aspartate aminotransferase, ALT - alanine aminotransferase, ALP - alkaline phosphatase, GGT - gammaglutamyl transferase 
Table 2. Radiological findings and duodenal tube aspiration test (DTA) in the studied groups

\begin{tabular}{|c|c|c|c|c|c|}
\hline \multirow{2}{*}{$\begin{array}{l}\text { Items } \\
\text { Liver span (cm) }\end{array}$} & \multicolumn{2}{|c|}{$\begin{array}{c}\text { BA } \\
(n=35)\end{array}$} & \multicolumn{2}{|c|}{$\begin{array}{c}\text { Non-BA } \\
(n=38)\end{array}$} & \multirow{2}{*}{$\begin{array}{l}P \text {-value } \\
0.470\end{array}$} \\
\hline & \multicolumn{2}{|c|}{$7.96 \pm 1.26$} & \multicolumn{2}{|c|}{$8.16 \pm 1.27$} & \\
\hline Splenic length $(\mathrm{cm})$ & \multicolumn{2}{|c|}{$6.34 \pm 1.23$} & \multicolumn{2}{|c|}{$6.18 \pm 1.47$} & 0.557 \\
\hline \multicolumn{5}{|l|}{ Polysplenia } & \multirow[t]{3}{*}{0.479} \\
\hline Present & 1 & $2.9 \%$ & 0 & $0 \%$ & \\
\hline Absent & 34 & $97.1 \%$ & 38 & $100 \%$ & \\
\hline \multicolumn{5}{|l|}{ Ascites } & \multirow[t]{3}{*}{0.916} \\
\hline Present & 3 & $8.6 \%$ & 3 & $7.9 \%$ & \\
\hline Absent & 32 & $91.4 \%$ & 35 & $92.1 \%$ & \\
\hline \multicolumn{5}{|l|}{ Gall bladder (GB) } & \multirow[t]{5}{*}{0.0001} \\
\hline Contractile & 4 & $11.4 \%$ & 28 & $73.7 \%$ & \\
\hline Non-contractile & 16 & $45.7 \%$ & 10 & $26.3 \%$ & \\
\hline Atretic & 12 & $34.3 \%$ & 0 & $0 \%$ & \\
\hline Non-visualized & 3 & $8.6 \%$ & 0 & $0 \%$ & \\
\hline \multicolumn{5}{|l|}{ Triangular cord sign (TC sign) } & \multirow[t]{3}{*}{0.029} \\
\hline Positive & 8 & $22.9 \%$ & 2 & $5.3 \%$ & \\
\hline Negative & 27 & $77.1 \%$ & 36 & $94.7 \%$ & \\
\hline \multicolumn{5}{|l|}{ Hepatic subcapsular flow } & \multirow[t]{3}{*}{0.001} \\
\hline Positive & 29 & $82.9 \%$ & 3 & $7.9 \%$ & \\
\hline Negative & 6 & $17.1 \%$ & 35 & $92.1 \%$ & \\
\hline \multicolumn{5}{|l|}{ DTA } & \multirow[t]{4}{*}{0.001} \\
\hline No bile stained secretions & 26 & $74.3 \%$ & 4 & $10.8 \%$ & \\
\hline Bile stained secretions & 0 & $0 \%$ & 3 & $8.1 \%$ & \\
\hline Not done & 9 & $25.7 \%$ & 31 & $81.1 \%$ & \\
\hline
\end{tabular}

infiltrate of (mild degree 56.3\%, moderate 25\%, and marked degree 9.4\%) but was not statistically different than that in the non-BA group. There was a statistically significant positive correlation between periductular neutrophils and other inflammatory reaction components including: acute cholangitis, portal cellular infiltrate $(p<0.01)$, mononuclear inflammatory cells in the ducts $(p<0.05)$, hepatocellular swelling $(p<0.01)$, pseudorosette formation and hepatocellular necrosis $(p<0.05)$ in the BA group.

Most of the BA group significantly had portal tract edema in liver tissues (91.4\% BA group vs. $39.5 \%$ in non-BA) ( $p<0.0001)$. Also the majority of the BA group showed either marked bridging (42.9\%) or focal porto-portal bridging (28.6\%), while the majority of the non-BA group showed grade 1 of portal fibrosis $(42.1 \%)(p<0.0001)$. Ductular proliferation of variable degrees was significantly more frequent in the BA group $(p<0.0001)$.

The presence of inflammatory cellular reactions (periductular neutrophils, mononuclear inflammatory cells in the ducts and pseudorosette formation) in liver tissues was significantly more frequent in the BA group than in the non-BA group $(p<0.05)$ together with visible bile plugs, ductular proliferation, portal tract edema, and higher grades of portal fibrosis, which were significantly able to diagnose BA with $57 \%$ sensitivity, $81 \%$ specificity, $74 \%$ PPV, 68\% NPV and 69\% accuracy in favor of the BA group $(p<0.0001)$.

\section{Cytokeratin 7 expression in liver tissues of the studied groups}

The majority of the BA group showed either grade 2 positive CK7 expression (a marker of biliary epithelium), in liver tissues (45.7\%) or grade 3 (34.3\%), while most of the non-BA group showed grade I $(71.1 \%)(p<0.01)$. Positive CK-7 expression in $>25 \%$ of the liver tissues had $91.4 \%$ sensitivity, $71.1 \%$ specificity, $74.4 \%$ PPV, $90.0 \% \mathrm{NPV}$ and $80.8 \%$ accuracy in discriminating between BA and non-BA in favor of BA. 


\section{VEGF expression in liver tissues of the studied groups}

There was statistically significant difference between BA and non-BA groups as regards VEGF expression in the portal structures (bile ducts [BDs] and arterial walls [AWs]) in liver tissues, where most of the BA group showed positive VEGF expression with variable degrees in each of the BDs $(80 \%)$ and $\mathrm{AWs}$ (77.2\%), while most of the non-BA group showed negative staining of VEGF in both BDs and AWs (89.5\% and $86.8 \%$ respectively) $(p<0.0001)$ (Table 3 ).

Positive VEGF expression in the portal structures in liver tissues in both BDs and AWs had 0.847, 0.820 area under ROC, $80.0 \%, 77.1 \%$ sensitivity, $89.5 \%$, $86.8 \%$ specificity, $87.5 \%, 84.4 \%$ PPV, $82.5 \%, 80.5 \%$ NPV and $84.9 \%, 82.19 \%$ accuracy respectively for BDs and AWs in discriminating between BA and non-BA $(p<0.0001)$.

The majority of the BA group showed grade 3 positive VEGF expression in zone 1,2 and 3 of the hepatocytes in liver biopsy ( $42.9 \%$ for each) $(p<0.05)$, while the majority of the non-BA group showed negative staining of VEGF in zone 1, 2 and 3 of the hepatocytes $(68.4 \%, 68.4 \%, 71.0 \%$ respectively) in liver tissues $(p<0.05)$ (Table 3). There was a significant positive correlation between VEGF expression in portal structures

Table 3. Grades of VEGF-A expression in bile duct (BD) wall in liver tissues of the studied groups

\begin{tabular}{|c|c|c|c|c|c|}
\hline $\begin{array}{l}\text { Grades } \\
\text { Grades of VEGF-A expression in bile ducts }\end{array}$ & \multicolumn{2}{|c|}{$\begin{array}{c}\text { BA } \\
(n=35)\end{array}$} & \multicolumn{2}{|c|}{$\begin{array}{l}\text { Non-BA } \\
(n=38)\end{array}$} & $\begin{array}{l}\text { P-value } \\
0.0001\end{array}$ \\
\hline No staining & 7 & $20 \%$ & 34 & $89.5 \%$ & \\
\hline Light yellow staining (grade 1) & 6 & $17.1 \%$ & 0 & 0 & \\
\hline Dark yellow staining (grade 2) & 7 & $20 \%$ & 0 & 0 & \\
\hline Brown staining with granularity (grade 3) & 15 & $42.9 \%$ & 4 & $10.5 \%$ & \\
\hline Grades of VEGF-A expression in wall of arterial blood vessels & & & & & 0.00063 \\
\hline No staining & 8 & $22.9 \%$ & 33 & $86.9 \%$ & \\
\hline Light yellow staining (grade 1) & 8 & $22.9 \%$ & 1 & $2.6 \%$ & \\
\hline Dark yellow staining (grade 2) & 7 & $20 \%$ & 0 & 0 & \\
\hline Brown staining with granularity (grade 3) & 12 & $34.2 \%$ & 4 & $10.5 \%$ & \\
\hline Grades of cytokeratin7 expression & & & & & 0.008 \\
\hline Grade $1(<25 \%)$ & 3 & $8.6 \%$ & 27 & $71 \%$ & \\
\hline Grade $2(25-50 \%)$ & 16 & $45.7 \%$ & 5 & $13.2 \%$ & \\
\hline Grade $3(50-75 \%)$ & 12 & $34.3 \%$ & 5 & $13.2 \%$ & \\
\hline Grade 4 (75-100\%) & 4 & $11.4 \%$ & 1 & $2.6 \%$ & \\
\hline Grades of VEGF expression in zone 1 hepatocytes & & & & & 0.009 \\
\hline No staining & 11 & $31.4 \%$ & 26 & $68.4 \%$ & \\
\hline Light yellow staining (grade 1) & 2 & $5.7 \%$ & 1 & $2.6 \%$ & \\
\hline Dark yellow staining (grade 2) & 7 & $20 \%$ & 2 & $5.3 \%$ & \\
\hline Brown staining (grade 3 ) & 15 & $42.9 \%$ & 9 & $23.7 \%$ & \\
\hline Grades of VEGF-A expression in zone 2 hepatocytes & & & & & 0.014 \\
\hline No staining & 12 & $34.3 \%$ & 26 & $68.4 \%$ & \\
\hline Light yellow staining (grade 1) & 1 & $2.9 \%$ & 1 & $2.6 \%$ & \\
\hline Dark yellow staining (grade 2) & 7 & $20 \%$ & 2 & $5.3 \%$ & \\
\hline Brown staining (grade 3) & 15 & $42.9 \%$ & 9 & $23.7 \%$ & \\
\hline Grades of VEGF-A expression in zone 3 hepatocytes & & & & & 0.019 \\
\hline No staining & 13 & $37.1 \%$ & 27 & $71 \%$ & \\
\hline Light yellow staining (grade 1) & 1 & $2.9 \%$ & 1 & $2.6 \%$ & \\
\hline Dark yellow staining (grade 2) & 6 & $17.1 \%$ & 2 & $5.3 \%$ & \\
\hline Brown staining (grade 3 ) & 15 & $42.9 \%$ & 8 & $21.1 \%$ & \\
\hline
\end{tabular}


(BDs and AWs) in liver tissues and VEGF in zone 1, 2, and 3 hepatocytes in both studied groups $(p<0.0001)$.

Most of the BA group who had positive VEGF expression in BDs (89.3\%) and AWs (92.6\%) in liver tissues also showed positive subcapsular hepatic flow, while most of the non-BA group who had negative VEGF expression in BDs (94.1\%) and AWs (93.9\%) in liver tissues showed negative subcapsular hepatic flow by color Doppler $(p<0.05)$. Also we found a significant positive correlation between positive subcapsular hepatic flow and positive VEGF expression in portal structures (BDs and AWs) in the BA group only $(p<0.05)$.

There was enlargement of the hepatic artery with a thickened medial layer in the BA group and some arteries showed a narrow to occluded lumen; however, there was no statistically significant difference between BA and non-BA groups $(p>0.05)$ as regards each hepatic artery diameter (HAD) $(998.2 \pm 946.84 \mu \mathrm{m}$ in the BA group vs. $902.9 \pm 816.39 \mu \mathrm{m}$ in non-BA) and medial thickness (MT) of the hepatic artery (188.58 \pm 114.33 $\mu \mathrm{m}$ in the BA group vs. $168.0 \pm 62.37 \mu \mathrm{m}$ in non-BA), but these levels were still higher in the BA group than non-BA. Also when comparing mean values of HAD and MT as regards VEGF expression; their values were higher in patients with positive VEGF in portal structures than those with negative VEGF expression but the results were not statistically significant $(p>0.05)$.

The degree of bile ductular proliferation had a significant positive correlation with $\mathrm{HAD}$ in the BA group $(p<0.001)$, while in the non-BA group it was significantly positively correlated with MT $(p<0.05)$. In addition we found that higher grades of portal fibrosis were significantly positively correlated with both $\mathrm{HAD}$ and MT in the non-BA group $(p<0.05)$.

In the non-BA group, there was a statistically significant positive correlation between VEGF-A expression in portal structures (BDs and $\mathrm{AWs}$ ) in liver tissues and the platelet count $(p<0.05)$. There was a statistically significant positive correlation between REMD and platelet count in both studied groups $(p<0.05)$.

In the current study, there was a statistically significant positive correlation between positive VEGF-A expression in portal structures in liver tissues with the degree of bile ductular proliferation $(p<0.05)$ in both studied groups, while there was a statistically significant positive correlation between positive VEGF-A in zone 1 and 2 hepatocytes with CK7 expression in liver tissues in the non-BA group only $(p<0.05)$.

In addition in this study we found that among cases with positive VEGF expression in portal structures, $50.0 \%$ of them had marked bridging fibrosis, and only one case $(3.1 \%)$ of them had absent or portal expansion of some portal tracts, while among those with negative VEGF in portal structures, $39.0 \%$ of them had absent or portal expansion of some portal tracts and only $9.8 \%$ had marked bridging fibrosis $(p<0.001)$. There was a statistically significant positive correlation between VEGF expression in portal structures in liver tissues and portal cellular infiltrate in the non-BA group $(p<0.05)$.

In the current study, most of the patients with successful Kasai had significant negative staining of VEGF (in AWs 66.7\%, BDs 55.6\% and hepatocytes 55.6\%) while most of those with failed Kasai had positive staining of VEGF (in AWs $91.3 \%$, BDs $91.3 \%$ and hepatocytes $86.9 \%)(p<0.05)$ (Table 4$)$.

\section{Discussion}

Although BA patients presented significantly earlier than the non-BA group, there was no significant difference between them as regards their mean age at admission. BA is difficult to identify during the perinatal period as few primary care physicians see more than 1 or 2 cases of BA during their careers, whereas neonatal jaundice (unconjugated hyperbilirubinemia) is extremely common, particularly among breast-fed infants [9] .

Serum ferritin and ALT were significantly higher in the non-BA group than in the BA group. Serum ferritin is a well-known inflammatory marker, but it is unclear whether serum ferritin reflects or causes inflammation, or whether it is involved in an inflammatory cycle [10]. Intrahepatic cholestasis is more related to hepatocellular damage [11], and it is not known if this elevated serum ferritin is a contributory factor for this cellular damage or it is released from damaged cells. The liver contains much of the iron stored in the body so any process that damages the liver cells will release ferritin [12].

The presence of more frequent bile plugs, ductular proliferation, periductular neutrophils, mononuclear inflammatory cells in the ducts, portal tract edema, pseudorosette formation and more severe grades of portal fibrosis in liver tissues was observed in the BA group. Lee et al. mentioned that, regardless of what might trigger an immune response included in the pathogenesis of BA, investigation of inflammatory substances might shed light on it. Such substances could theoretically be useful markers of disease risk or even potential therapeutic targets [13].

The majority of the BA group showed higher grades of positive CK7 expression in liver tissues, while most of the non-BA group showed lower grades. Positive CK7 expression in $>25 \%$ of the liver tissues had a very good accuracy (80.8\%) in discriminating between BA and non-BA in favor of BA. 
Table 4. Preoperative VEGF-A expression in liver tissues from BA patients with successful vs. failed Kasai

\begin{tabular}{|c|c|c|c|c|c|}
\hline $\begin{array}{l}\text { VEGF-A } \\
\text { VEGF-A in BDs }\end{array}$ & \multicolumn{2}{|c|}{$\begin{array}{c}\text { Successful Kasai } \\
n=9(28.1 \%)\end{array}$} & \multicolumn{2}{|c|}{$\begin{array}{c}\text { Failed Kasai } \\
n=23(71.9 \%)\end{array}$} & \multirow{2}{*}{$\begin{array}{c}P \text {-value } \\
0.012\end{array}$} \\
\hline No staining & 5 & $55.6 \%$ & 2 & $8.7 \%$ & \\
\hline Light yellow & 0 & $0 \%$ & 6 & $26.1 \%$ & \\
\hline Dark yellow & 2 & $22.2 \%$ & 3 & $13.0 \%$ & \\
\hline Brown with granularity & 2 & $22.2 \%$ & 12 & $52.2 \%$ & \\
\hline VEGF-A in AWs & & & & & 0.005 \\
\hline No staining & 6 & $66.7 \%$ & 2 & $8.7 \%$ & \\
\hline Light yellow & 0 & $0 \%$ & 7 & $30.4 \%$ & \\
\hline Dark yellow & 1 & $11.1 \%$ & 4 & $17.4 \%$ & \\
\hline Brown with granularity & 2 & $22.2 \%$ & 10 & $43.5 \%$ & \\
\hline VEGF-A in zone I hepatocytes & & & & & 0.042 \\
\hline No staining & 5 & $55.6 \%$ & 3 & $13.0 \%$ & \\
\hline Light yellow & 0 & $0 \%$ & 2 & $8.7 \%$ & \\
\hline Dark yellow & 0 & $0 \%$ & 7 & $30.4 \%$ & \\
\hline Brown with granularity & 4 & $44.4 \%$ & 11 & $47.8 \%$ & \\
\hline VEGF-A in zone II hepatocytes & & & & & 0.079 \\
\hline No staining & 5 & $55.6 \%$ & 4 & $17.4 \%$ & \\
\hline Light yellow & 0 & $0 \%$ & 1 & $4.3 \%$ & \\
\hline Dark yellow & 0 & $0 \%$ & 7 & $30.4 \%$ & \\
\hline Brown with granularity & 4 & $44.4 \%$ & 11 & $47.8 \%$ & \\
\hline VEGF-A in zone III hepatocytes & & & & & 0.163 \\
\hline No staining & 5 & $55.6 \%$ & 5 & $21.7 \%$ & \\
\hline Light yellow & 0 & $0 \%$ & 1 & $4.3 \%$ & \\
\hline Dark yellow & 0 & $0 \%$ & 6 & $26.1 \%$ & \\
\hline Brown with granularity & 4 & $44.4 \%$ & 11 & $47.8 \%$ & \\
\hline
\end{tabular}

N.B. Two cases of the BA group were lost to follow-up before determining the diagnosis, and one case died postoperatively due to surgical causes.

The findings of our results that there is higher expression of VEGF-A in zone 1,2 and 3 of the hepatocytes and portal structures (BDs and AWs) in liver biopsy of the BA group than that of the non-BA group may be explained by the presence of arteriopathy in branches of the hepatic artery in the extrahepatic biliary tree of patients with BA. It has been proposed that the vasculopathy may be the primary lesion in patients with BA [14].

Edom et al. found that, in BA at the time of portoenterostomy, VEGF was positive in AWs and BDs, besides hepatocytes. He explained that, in BA, there is portal expansion, and the mechanical distortion over vascular structures may induce hypoxia/ischemia. The mechanical distortion may be caused by extracellular matrix deposition that offers resistance to blood flow, inducing hypoxia and VEGF-A positive expression in the portal structures [5].
Uflacker and Parinte found out that in BA, there is early arteriographic evidence of dystrophic peripheral hepatic artery branches, with irregularities in contour and images suggestive of occlusion. They reported it as an angiographic vascular "tuft-like" blush surrounding occluded peripheral arterial segments [15].

In addition Lee et al. found that, on color Doppler US, an enlarged hepatic artery and hepatic arterial flow that extended to the hepatic surface were seen in all patients with BA [16]. EL-Guindi et al. reported that hepatic subcapsular flow was found in most of the BA group compared with the non-BA group and in none of the non-hepatic control group $(p<0.0001)$ [17].

The relation between the simultaneous increased expression of VEGF-A in BDs and AWs in liver tissues as well as presence of subcapsular hepatic flow in the BA group, and absence of this relation in the non-BA 

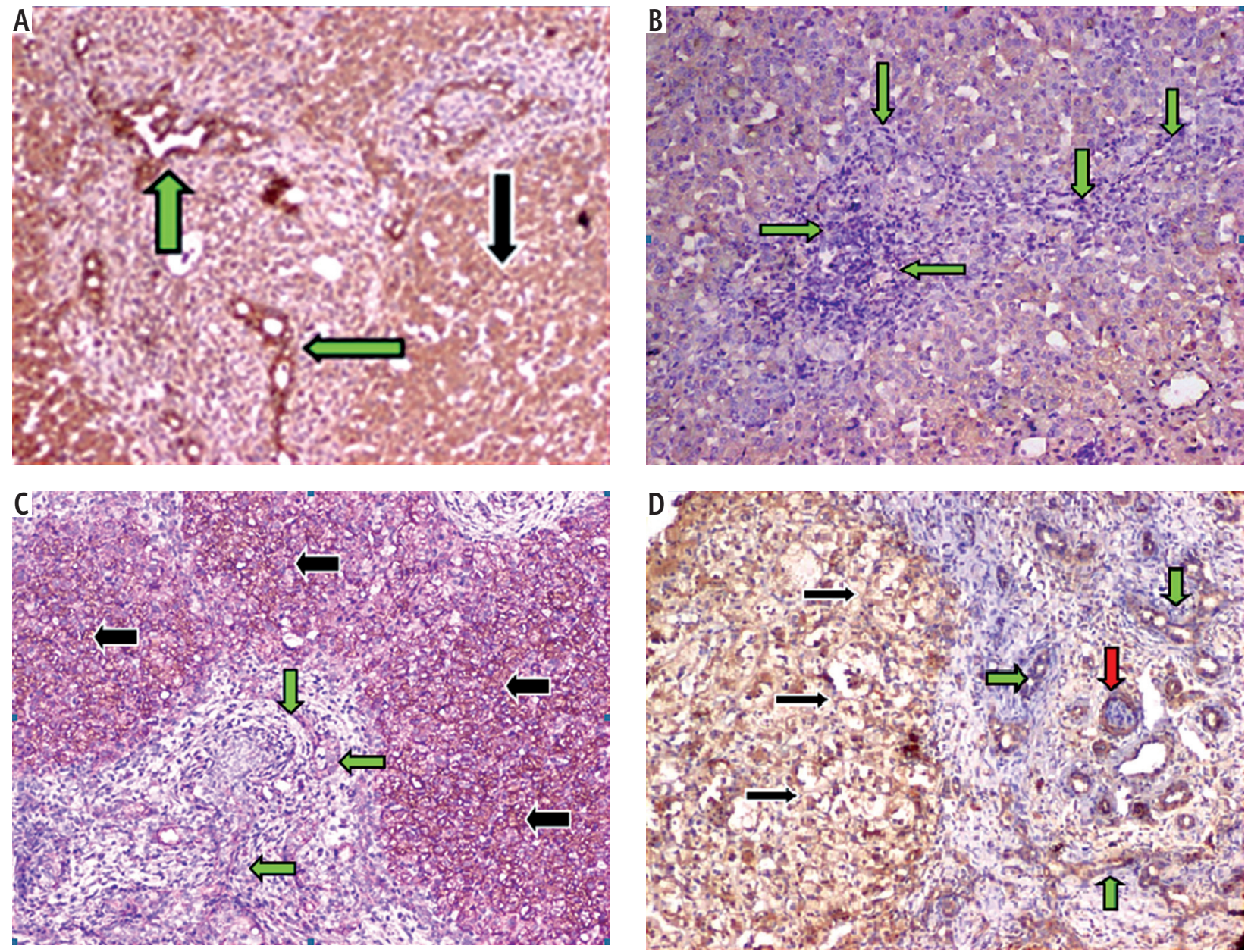

Fig. 1. A) These figures show positive VEGF in a case of a female infant 61 days old with type-III BA. There was brown positivity of VEGF in hepatocytes (black arrows), and in bile duct wall with stronger intensity (green arrows), magnification 100x. B) Negative staining of VEGF in 50-day-old male infant with successful post-Kasai in liver tissues. C) Positive VEGF in a case of a male infant 180 days old with CHF in liver tissues. There was very light brown color of positive VEGF expression in periductal (green arrows) compared to dark brown staining in hepatic parenchyma (black arrows), magnification 10x. D) Positive VEGF expression in 3-month-old male infant with PFIC-III in liver tissues. There was brown color in hepatocytes (black arrow), peri-ductal (green arrows) and periarterial blood vessel (red arrow), magnification 100x

group in the current study supports the putative role of an arteriopathy in the development of BA [18].

The significant positive correlation between VEGF-A expression in portal structures (BDs and AWs) in liver tissues and the platelet count in the nonBA group is supported by the Chongsrisawata et al. report that platelets engulf and store various angiogenic factors such as VEGF and platelet-derived growth factor (PDGF) in their granules, and these molecules are secreted immediately after platelet activation [19].

The fibrogenic effect of VEGF through multiple mechanisms, including promotion of inflammation, release of fibrosis-enhancing molecules from VEGF activated endothelial cells, and direct effects of VEGF on HSC [20], can explain the significant positive correlation between VEGF-A expression in portal structures and the degree of bile ductular proliferation in both groups.
So first we have a stimulus (whatever its source and quality) that would cause damage to biliary epithelia with subsequent events including cross talk between cholangiocytes proliferation and angiogenesis in the presence of inflammatory chemokines and the cytokine environment that would upregulate VEGF expression continuing through a vicious circle of increased cholangiocyte proliferation, VEGF secretion, and local inflammatory recruitment ending in fibrosis.

Sokol and Mack explained these events in which chemokines and adhesion molecules released by cholangiocytes and vascular endothelial cells recruit local macrophages and neutrophils that then release TNF- $\alpha$, nitric oxide, and other reactive oxygen species [21]. The VEGF-mediated cross talk between hepatic progenitor cells (HPC)/ductular reaction (DR) and endo- 
thelial cells may be involved in the remodeling of the vascular bed occurring in the ductular reaction [22].

Presence of negative staining of VEGF-A in most of the patients with successful Kasai and positive staining in most of those with failed Kasai highlights the idea of using VEGF inhibitors in BA disease and bringing such concepts from bench to bedside. Friedman (2008) stated that VEGF inhibitors are an effective anti-inflammatory and antifibrotic strategy, but their longterm impact on regeneration is uncertain [23].

In conclusion, VEGF-A expression in the portal structures in liver tissues in both BDs and AWs had very good accuracy in discriminating between BA and non-BA patients.

\section{Disclosure}

The authors report no conflict of interest.

\section{References}

1. Santos JL, Carvalho E, Bezerra JA. Advances in biliary atresia: from patient care to research. Braz J Med Biol Res 2010; 43: 522527.

2. Deltenre P, Valla DC. Ischemic cholangiopathy. J Hepatol 2006; 44: 806-817.

3. Kantari-Mimoun C, Castells M, Klose R, et al. Resolution of liver fibrosis requires myeloid cell-driven sinusoidal angiogenesis. Hepatology 2015; 61: 2042-2055.

4. Yancopoulos GD, Davis S, Gale NW, et al. Vascular-specific growth factors and blood vessel formation. Nature 2000; 407: 242-248.

5. Edom PT, Meurer L, da Silveira TR, et al. Immunolocalization of VEGF $A$ and its receptors, VEGFR1 and VEGFR2, in the liver from patients with biliary atresia. Appl Immunohistochem Mol Morphol 2011; 19: 360-368.

6. Glaser SS, Gaudio E, Alpini G. Vascular factors, angiogenesis and biliary tract disease. Curr Opin Gastroenterol 2010; 26 : 246-250.

7. Wu AW, Gu J, Li ZF, et al. COX-2 expression and tumor angiogenesis in colorectal cancer. World J Gastroenterol 2004; 10: 2323-2326

8. Yabushita K, Yamamoto K, Ibuki N, et al. Aberrant expression of cytokeratin 7 as a histological marker of progression in primary biliary cirrhosis. Liver 2001; 21: 50-55.

9. Powell JE, Keffler S, Kelly DA, Green A. Population screening for neonatal liver disease: potential for a community-based programme. J Med Screen 2003; 10: 112-116.

10. Kell DB, Pretorius E. Serum ferritin is an important inflammatory disease marker, as it is mainly a leakage product from damaged cells. Metallomics 2014; 6: 748-773.

11. Gupta NA, Karpen SJ. Mechanisms of bile formation and cholestasis. In F. J. Suchy, R. J. Sokol, W. F. Balistreri (eds.). Liver disease in children. Cambridge University Press, United Kingdom 2014; 24-31.

12. Linder MC. Mobilization of stored iron in mammals: a review. Nutrients 2013; 5: 4022-4050.

13. Lee HC, Chang TY, Yeung CY, et al. Genetic variation in the vascular endothelial growth factor gene is associated with biliary atresia. J Clin Gastroenterol 2010; 44: 135-139.
14. Sokol RJ, Shepherd RW, Superina R, et al. Screening and outcomes in biliary atresia: summary of a National Institutes of Health workshop. Hepatology 2007; 46: 566-581.

15. Uflacker R, Pariente DM. Angiographic findings in biliary atresia. Cardiovasc Intervent Radiol 2004; 27: 486-490.

16. Lee MS, Kim MJ, Lee MJ, et al. Biliary atresia: color doppler US findings in neonates and infants. Radiology 2009; 252: 282-289.

17. El-Guindi MA, Sira MM, Konsowa HA, et al. Value of hepatic subcapsular flow by color Doppler ultrasonography in the diagnosis of biliary atresia. J Gastroenterol Hepatol 2013; 28: 867-872.

18. de Souza AF, Meurer L, da Silveira TR, et al. Angiopoietin 1 and angiopoietin 2 are associated with medial thickening of hepatic arterial branches in biliary atresia. Pediatr Res 2014; 75: 22-28.

19. Chongsrisawata V, Vejchapipatb P, Poovorawana Y. Serum vascular endothelial growth factor per platelet count in patients with biliary atresia. Asian Biomedicine 2010; 4: 223-229.

20. Sahin H, Borkham-Kamphorst E, Kuppe C, et al. Chemokine Cxcl9 attenuates liver fibrosis-associated angiogenesis in mice. Hepatology 2012; 55: 1610-1619.

21. Sokol RJ, Mack CL. Biliary atresia and the ductal plate. In: K. F. Murray (ed.). Clinical gastroenterology: fibrocystic diseases of the liver. Vol. 8. Springer Science + Business Media, LLC 2010; 179-199.

22. Spirli C, Strazzabosco M. Vascular endothelial growth factors in progenitor cells mediated liver repair. Hepatobiliary Surg Nutr 2013; 2: 65-67.

23. Friedman SL. Mechanisms of hepatic fibrogenesis. Gastroenterology 2008; 134: 1655-1669. 